\title{
Reflections on 40 years as a sideline physician
}

\author{
G. ROBERT Nugent, M.D. \\ Department of Neurosurgery, The Robert C. Byrd Health Sciences Center of West Virginia University, \\ Morgantown, West Virginia
}

\begin{abstract}
Object. In this study the author presents his personal observations concerning football injuries in a historical perspective with additional literature citations.

Methods. Aspects of brachial plexus and cervical spine injuries, neurapraxia, face mask infractions, concussion, acute subdural hematoma (SDH), and the so-called second-impact syndrome are addressed.

Conclusions. The list of conclusions presented in this paper is as follows: there is more than one kind of brachial plexus injury; wedging of cervical vertebrae may be normal; neurapraxia presents a problem for return to play; face mask injuries are rarely serious; definitions of concussion vary; acute SDH requires immediate transfer to a hospital; and the second-impact syndrome may be a myth to some.
\end{abstract}

\section{KEY WORDS • brachial plexus injury • neurapraxia • concussion • subdural hematoma • second-impact syndrome}

I N 1967, after taking care of a football player who would now be considered to have had the condition called neurapraxia, I was asked by the West Virginia football coach if I would be willing to sit on the bench at home games. Over time, this involvement expanded to the point that I was traveling with the team when it was convenient for me. A lasting interest in football and its injuries became part of this experience. Along the way certain observations emerged, which I offer in this article, along with literature support, to those who have not enjoyed as extensive an experience in the field.

\section{Brachial Plexus Injuries}

Unquestionably the most common neurological injury to be seen on the sidelines is the "stinger," which is most often a brachial plexus stretch injury. Players who suffer this injury take themselves off the field, usually in severe pain and often holding the affected arm in some degree of pronation or supination. Many experience this as a recurring but transient injury. It usually occurs from an impact that drives the shoulder down and the head away from the shoulder. When the player is asked which fingers are numb, the answer almost invariably is: "All the fingers, Doc." This implies an injury to at least the C-6, -7 , and -8 roots. Most of the time there is no associated weakness, and the athlete is usually pain free in 15 or 20 minutes and can return to play. If weakness is present, it is interesting that it is almost always limited to the deltoid and to some degree the biceps muscle. This implies upper plexus or C-5 root involvement and a paradoxical discrepancy with the sensory root pattern. The most important consideration for the

Abbreviations used in this paper: $\mathrm{SDH}=$ subdural hematoma; $\mathrm{VB}=$ vertebral body. sideline examiner is to check for deltoid weakness, which would be the limiting factor for return to play. When weakness is present, an electromyography study should be performed at least 3 weeks after the injury but, as often as not, the weakness will have resolved by that time.

Although classically and usually this is a plexus problem related to the shoulder being displaced down while the head is displaced in the opposite direction, occasionally a similar pain pattern occurs when the inner edge of the shoulder pads is driven down onto the upper brachial plexus at the base of the neck. Two findings implicate this injury: 1) there is exquisite tenderness to pressure on the trapezius muscle; and 2) often, if weakness is also present, there is sparing of the rhomboid muscles. The nerve to the rhomboids branches off the plexus proximal to the site of injury (Fig. 1). ${ }^{5}$ Weakness of the rhomboids can be detected by having the player, with his hand on his hip, displace the elbow posteriorly against resistance while the examiner palpates the rhomboid muscles between the shoulder blades with the other hand (Fig. 2). When this is the problem, shoulder pads can usually be modified by the equipment manager to prevent recurrence, often by the application of extra padding to eliminate the dead space between the shoulder pad and the hypertrophied trapezius muscle.

One linebacker on our team had severe, recurring weakness of his deltoid and biceps muscles. The worry was that recurrent injury might lead to permanent weakness. For supportive advice (not wanting to terminate him from play myself because he was one of our most outstanding players) I referred him to my friend Joe Maroon in nearby Pittsburgh, who thought that the problem was serious enough that the linebacker should no longer play. We could not bring ourselves to terminate this outstanding player so, as a trial, we placed him on a rigorous program of exercises to strengthen his neck, which he performed throughout the summer. He increased his neck circumference by 2 in, 


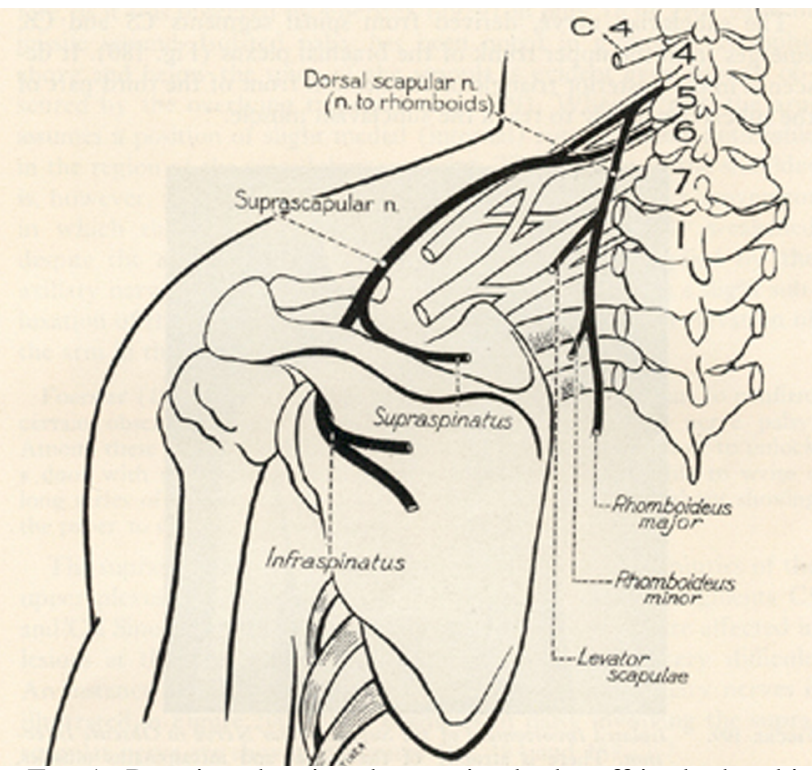

FIG. 1. Drawing showing the proximal take-off in the brachial plexus of the nerve (n) to the rhomboid muscles. Reproduced with permission from Elsevier; from Haymaker W and Woodhall B: Peripheral Nerve Injuries: Principles of Diagnosis. Philadelphia: WB Saunders, 1953, p 229.

continued to play without further difficulty, and became a consensus all-American, playing many years in the National Football League. Such a program should be a consideration whenever a similar situation arises.

\section{Cervical Spine Injuries}

These days, with any suspicion or complaint of a neck injury or pain, $\mathrm{x}$-ray films of the cervical spine are routinely obtained to clear and protect the player. These are often performed after very minimal injury in an overly protective fashion, with the team physician realizing that it is highly unlikely that the films will show any abnormality. An occasional complicating factor relates to the radiologist reading the films as showing, for example, "a compression fracture of C-5." This result can be catastrophic, because suddenly trainers, doctors, and especially coaches have a "broken neck" on their hands. At the same time, everyone involved has a firm belief that the injury could not have been that severe. It is not known to all, including some radiologists, that wedging of the C-5 and other VBs can be present as an incidental, normal radiographic variant. Keats, ${ }^{6}$ in a text on normal radiographic variations, shows this type of wedging (Fig. 3). A bone scan was often necessary to establish that the injury was not acute, but today a skilled radiologist with a good magnetic resonance image available for review can determine that this is not an acute injury.

\section{Experiences With Neurapraxia}

Although the term "neurapraxia" has been used traditionally to describe transient loss of motor and sensory function without axonal injury (as seen with peripheral nerve injuries), it has more recently been used to describe the transient spinal cord dysfunction associated with cervical injury. This aspect has been most studied and written about by the orthopedist Joe Torg. ${ }^{12}$ More recently, Dr. Bailes ${ }^{1}$ has also discussed this in the neurosurgical literature.

According to Torg's studies, the entity takes the following forms, with a transient quadriplegia being the most common. His classification system is as follows: Type 1, plegia (complete paralysis); Type 2, paresis (motor weakness); and Type 3, paresthesias (sensory changes without motor involvement). Duration of deficits: Grade 1, fewer than 15 minutes; Grade 2, 15 minutes to 24 hours; and Grade 3, longer than 24 hours. Pattern of deficits: Pattern 1, quad (all four extremities involved); Pattern 2, upper (both arms involved); Pattern 3, lower (both legs involved); and Pattern 4, hemi (ipsilateral arm and leg involved).

Almost always the problem relates to the fact that these players have a congenitally narrow cervical canal in the anteroposterior dimension. With this predisposition, the spinal cord is vulnerable to hyperextension injuries, which cause inbuckling of the ligamentum flavum and cord compression. ${ }^{4,7}$ This may be compounded by the coexistence of a bulging disc. The narrow canal can be demonstrated by the Torg ratio, which is used to compare the width of the VB with that of the spinal canal. ${ }^{12} \mathrm{~A}$ ratio of 0.8 or less confirms a narrow canal. This has been disputed, however, by those who note that VBs in football players, like the rest of their bodies, are larger than normal, thus distorting the ratio. Nevertheless, a narrow canal is the common observation in others' experience, as well as my own.

The existence of a narrow canal can be readily appreciated on plain lateral cervical spine x-ray films. A significant space between the shadow of the facet and the line of the lamina indicates a wide or normal canal. When the shadow of the lamina is close to or up against the facet, the canal is congenitally narrow (Fig. 4). In a markedly narrow

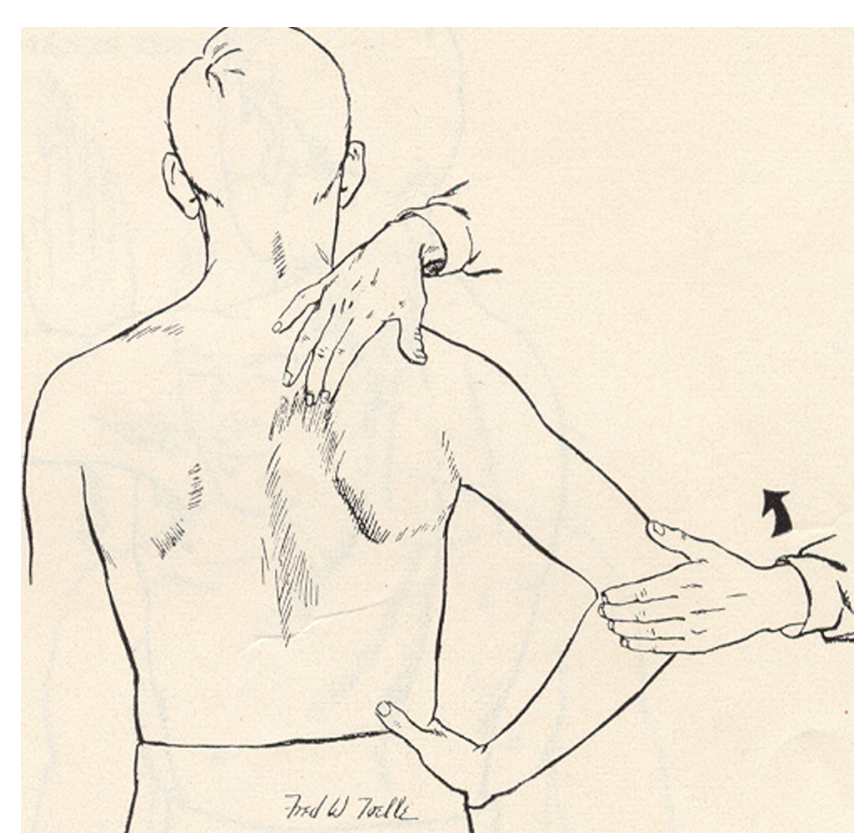

FIG. 2. Drawing showing the method for checking the strength of the rhomboid muscles. Reproduced with permission from Elsevier; from Haymaker W and Woodhall B: Peripheral Nerve Injuries: Principles of Diagnosis. Philadelphia: WB Saunders, 1953, $\mathrm{p} 75$. 


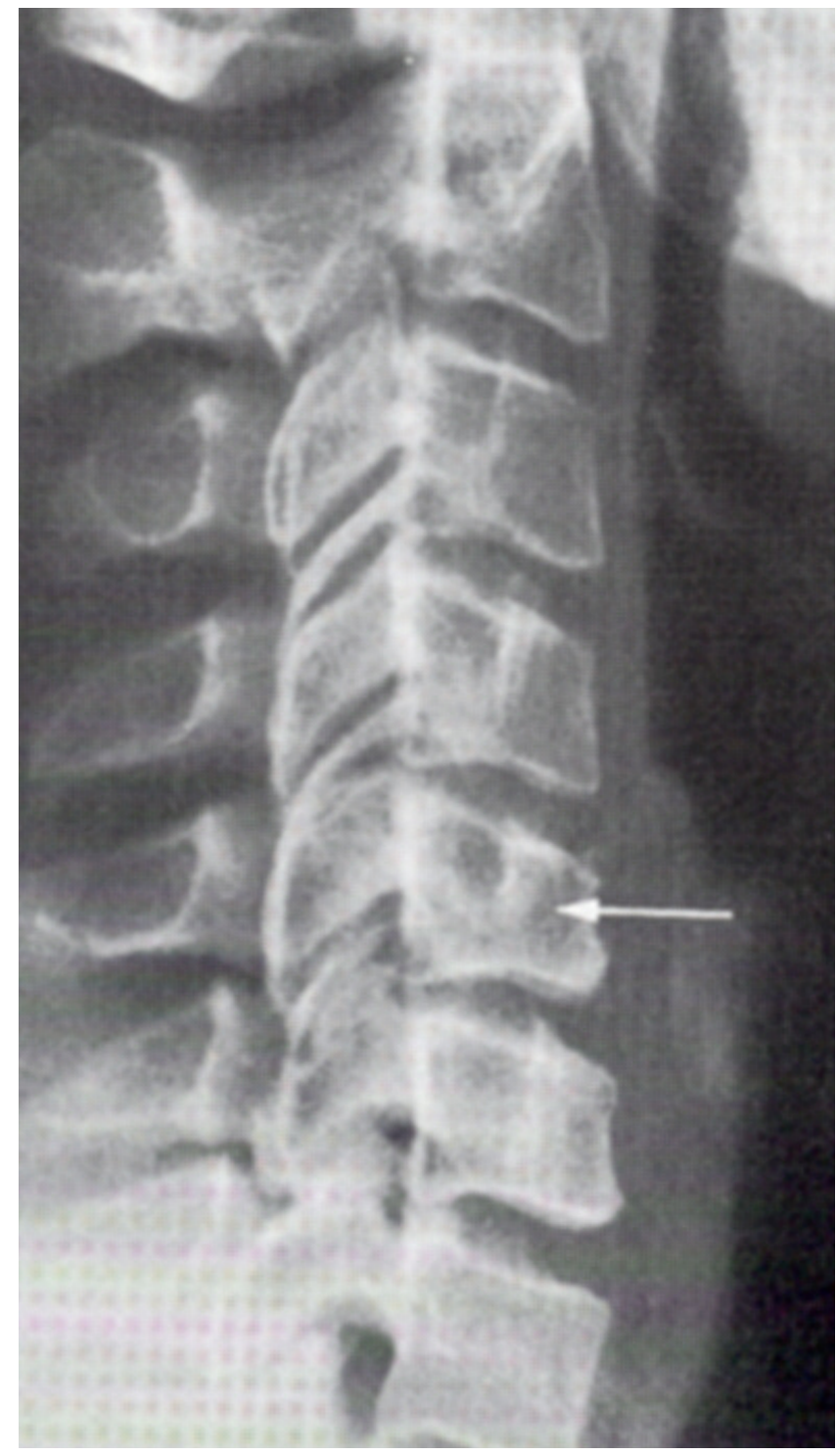

FIG. 3. An x-ray film showing normal wedging of the C-5 VB (arrow) Reproduced with permission from Elsevier; from Keats TE: Atlas of Normal Roentgen Variants that May Simulate Disease, 6th ed. St. Louis: Mosby, 1996, p 208.

canal, the line of the lamina is superimposed on that of the facet. This radiographic finding of a narrow canal is also the underlying problem in the following conditions: symptomatic cervical spondylosis, the central cord syndrome seen with hyperextension injuries, and with ossification and hypertrophy of the posterior longitudinal ligament that has become symptomatic.

There has been a dispute, largely between neurosurgeons and orthopedists, whether a player with neurapraxia should be allowed to continue playing football. From their study of 100 of these patients, Dr. Torg et al., ${ }^{12}$ believe that this injury does not portend a more serious one, and that the player can continue to play, but only with the knowledge that the injury may recur. I think that neurosurgeons in general take a more serious attitude and consider quadriplegia, even when transient, as a threatening and limiting injury.
The paralysis may be very short-lived: 15 to 20 seconds or a few minutes in duration. In recent years, if this is the case, I have permitted these players to return to the field and have noted no adverse effects. Dr. Bailes ${ }^{1}$ has also returned some to play who had very brief deficits. In my years as a team physician I have terminated the participation of players with this problem at least eight or nine times. This is often a difficult decision, but it is sometimes aided by the family of the player (usually the mother, who decides that her boy should not further expose himself to this risk).

\section{Face-Mask Penalty}

The face-mask penalty has been with us now for many years. It was perhaps first brought to the attention of neurosurgeons by Dr. Richard Schneider, ${ }^{11}$ who had a long-standing interest in sports injuries to the nervous system. Dr. Schneider's dramatic illustrations in his book emphasized the rather extreme degree of head and neck involvement associated with this infraction (Fig. 5). He has also shown a player whose head was rotated $180^{\circ}$ from normal and whose body was subsequently spun around $360^{\circ}$. The original caption on this illustration indicated that no injury occurred. I have witnessed the most severe face-mask violations imaginable (players spun $360^{\circ}$ by an opposing player holding the face mask, players slung to the ground by an opponent grabbing the face mask, and worse). However, the interesting observation is that this rarely leads to injury and the player doesn't usually even leave the game. At various conferences of team physicians, trainers, and so forth, I usually ask for a show of hands from those who have ever seen a significant injury occur because of a face-mask violation. No one ever raises a hand. I would be grateful to learn from others if they have seen such an injury; I suspect that they do occur. I don't believe this penalty should be removed, but I do think that the violation has been exaggerated as a problem and is more feared than it should be. I also think that the automatic 5-yard penalty for just touching the face mask is a pure gift and should be eliminated.
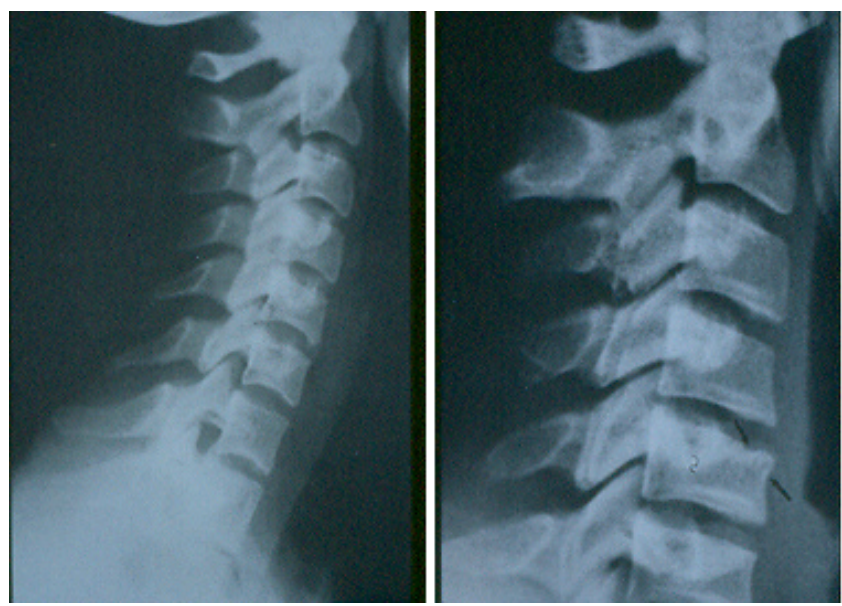

Fig. 4. Cervical x-ray films showing different spinal canal widths. Left: A wide canal in the sagittal dimension, as shown by the space between the back of the facet and the line of the lamina. Right: A narrow canal, as depicted by the line of the lamina, which is only narrowly separated from the back of the facet. 


\section{Concussion}

Attitudes about concussion as seen in football have changed dramatically over the past 40 years. In the 1960s, we had no guidelines for concussion. With the now-antiquated helmets of that time, concussion was far more common than today, but it was taken much less seriously. It would not be unusual for a player who was "dinged" to be told to "suck it up" and return to play. It also would not be unusual for a player to tell you that he didn't remember anything until the afternoon of the day after the injury, and he would be permitted to return to play at that time. Today, this degree of retrograde amnesia would keep him out of play until he had been asymptomatic for 1 week. I must say that I do not remember any serious problems that arose as a result of returning players to the game or practice with injuries that today would limit that participation.

There are now a dozen or more classifications of and grading scales for concussion, which leads to some confusion about the value of these classifications, particularly as they relate to decisions on whether an athlete may return to play. The most minimal expression of concussion is confusion. A somewhat worse injury involves some degree of amnesia, and if it is worse still, unconsciousness results. Several features of concussion exist that may be more helpful in recognizing this entity than the classifications currently in use. These include the following: a detached or vacant facial expression; inappropriate responses and irrelevant statements; the repeating of statements and questions; unexpected emotional reactions such as crying; a delay in responding to commands; or an unrealistic assurance that all is "OK." More obvious problems include disorientation, difficulty reversing a series of digits and serial subtractions, and evidence of amnesia.

\section{Acute SDH}

This acute process, which is the most feared of sports injuries, usually progresses rapidly because the underlying cause is cortical arterial bleeding, which causes a rapid development of coma in some cases. These are players whose injury may progress from head trauma to tentorial herniation within 1 hour. In his book, ${ }^{11}$ Dr. Schneider describes a player who sustained a head injury while scrimmaging. The player quickly fell over unconscious and a dilated pupil developed soon thereafter. He was immediately transported to a hospital, whereupon his pupils became bilaterally dilated and fixed. One hour after the injury, the SDH was evacuated, and the player made a complete recovery. A second case was described with a similar story; a football-related head injury followed by a dilated pupil. The SDH was removed 1.5 hours after the injury and the patient made a complete recovery. The point is that the team physician must be aware of the possibility of rapid deterioration and also of the logistics of the situation so that he or she can personally assure that such a patient is rapidly transferred to a neurosurgical facility, calling ahead so that the staff is prepared to act immediately.

After an experience at an "away" game where the team buses could not get out of a gate-enclosed area for almost 1 hour (because someone had failed to arrange for the gate to be unlocked after the game), it occurred to me that if I were responsible for the acute management of one of our

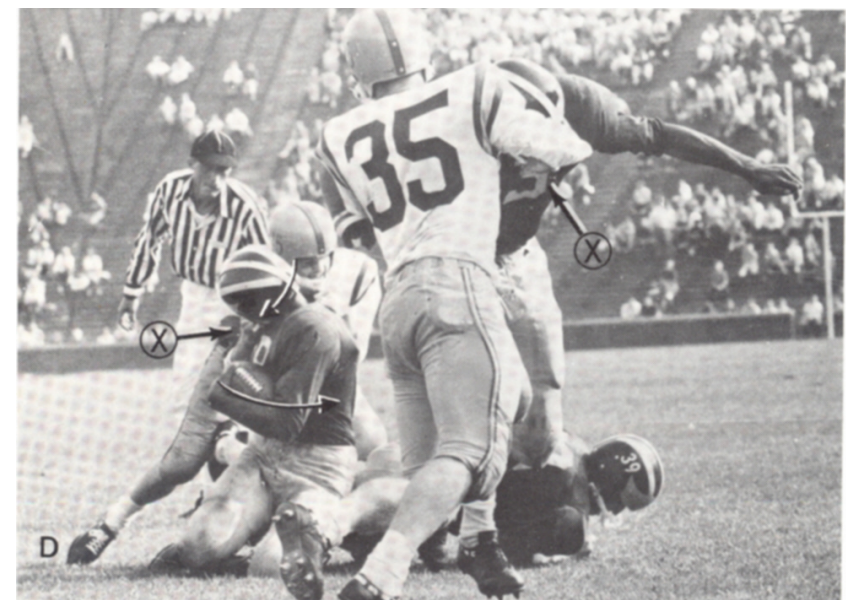

FIG. 5. Photograph taken during a game, showing an apparently severe face-mask violation. The player was described as suffering no injury. Reproduced with permission from Schneider RC: Head and Neck Injuries in Football: Mechanisms, Treatment, and Prevention. Baltimore: Williams and Wilkins, 1973, p 99.

players in a similar situation in which emergency evacuation was impossible, I should be prepared to handle the problem myself. This led to my practice of carrying a small bur hole tray with me to away games. This did not last, however, because it soon became apparent that a trauma of this nature often has dire outcomes, which could lead to serious medicolegal issues. I could be sued for practicing medicine out of state without a license.

It is hard to believe that because of poor planning and foresight, a football player (or a boxer rendered unconscious at an arena in a major city) cannot be transported to a local hospital for emergency surgery in time to prevent death or a disastrous outcome. There must be an organized plan and a knowledgeable person on hand to take over and forcefully guide the situation, getting the injured person to surgery expeditiously.

\section{The Second-Impact Syndrome}

In 1984 Saunders and Harbaugh ${ }^{10}$ described a football player who after a head injury remained symptomatic for 1 week but still returned to play, only to suffer a second injury with edema, which was fatal. It was postulated that the cause of the fatal cerebral hemorrhage was a failure of autoregulation. This was the birth of the so-called secondimpact syndrome, about which much concern and fear has been expressed ever since. Although I have not seen the syndrome, I have had a long-standing interest in the matter and have had some reservations about its validity. If it is a real problem, why don't we see it regularly in boxers? And what is the evidence that this phenomenon is due to a failure of autoregulation?

In 1998, McCrory and Berkovic ${ }^{8}$ reviewed the reported cases of this syndrome and found only 17 that met their criteria for the entity. Their criteria consisted of the following: 1) medical review after a witnessed first impact; 2) documentation of ongoing symptoms following the initial impact up to the time of the second impact; 3 ) witnessed second head impact with a subsequent rapid cerebral deterioration; and 4) neuropathological or neuroimaging evidence of cerebral swelling without a significant intracra- 
nial hematoma or another cause of cerebral edema (for example, encephalitis). They found that none of the reported cases met these criteria and in 12 of them there was no documented second impact; many of the players merely collapsed during the second play. There were only five probable cases of the syndrome, and the athletes involved included three boxers, one ice hockey player, and one high school football player. It was of interest that all of these probable cases, and most of the ones in the series, involved teenagers. McCrory and Berkovic raise the question whether the second-impact syndrome really exists or whether it is more related to the severe and often fatal cerebral edema seen in association with head trauma in children, which has been described previously. ${ }^{2,3,9}$ Is this problem then unrelated to a second impact, and is it one that is more of a first-impact syndrome that is unique to injury in the young brain?

\section{Other Observations}

Playing experience is of utmost importance to the player who foresees a possible career in professional football. At times this issue may confuse the situation because the player will minimize his injury so that he can continue to play. That a deception is being played out is often most apparent to the trainers, who have the closest contact with the player, and can help clear the air in this regard.

Another occasional problem is a parent (usually the father) who has a determined interest in having his son continue to play despite an injury or a situation that is potentially a serious risk for the athlete. As stated earlier, the mother is often in control and wisely makes the decision not to let her son play. Sometimes, though, if he is not satisfied with the opinion of the team physician and others, the father may take the young man to various team doctors around the country, hoping that one will say that his son can play. So far, the threatening nature of the underlying problem has been obvious and the athlete has not found a doctor who would release him, and we have therefore not found ourselves in an embarrassing situation.

Despite regulations, admonitions by coaches, and a prevailing negative attitude toward the use of alcohol during the playing season, this is a common violation. When examining a player in the locker room Sunday morning after a Saturday game, the odor of alcohol is often obvious. This after-game celebration is almost a way of life for some and one that is difficult to control. It does seem, however, that this rarely interferes with the players' performances come game time.

In my earlier experience, hysterical reactions were not uncommon in the football player. A player, for instance, who had missed his assignment, letting the opponent make a substantial gain, might come off the field in a confused and dazed state to create the impression that because of a "head injury" he wasn't responsible for his actions. No longer do we see feigned reactions to justify a failed performance. Just as hysteria is generally less common in the population at large, the modern young man seems to be more mature and more sophisticated and does not resort to these techniques.

\section{Conclusions}

In this article I have attempted to clarify the following issues involving football-related injuries: 1) the brachial plexus stretch injury and its variations, with emphasis on the disparity between the sensory and motor findings and shoulder pad injuries to the plexus that may mimic the more common proximal injury; 2) lack of awareness that wedging of the cervical VBs may be a normal variant and is not indicative of an acute injury; 3 ) the controversy related to return to play after transient quadriplegia as seen with neurapraxia, with discussion of the prominence of a narrow spinal canal in the sagittal dimension as a causative factor; 4) the argument that the face-mask violation rarely causes a significant injury; 5) the varieties and vagaries of concussion, with suggestions for the on-field evaluation of the player who suffers this injury; 6) acute SDH being one of the most serious injuries requiring good logistical planning and action for immediate management; and 7) the idea that second-impact syndrome may not be a specific clinical injury. The cases reported have not met the criteria for this entity, and some think that the condition is something of a myth. This is not to say, however, that the symptomatic athlete should be returned to play.

\section{References}

1. Bailes JE: Experience with cervical stenosis and temporary paralysis in athletes. J Neurosurg Spine 2:11-16, 2005

2. Bruce DA: Delayed deterioration of consciousness after trivial head injury in childhood. Br Med J (Clin Res Ed) 289:715-716, 1984

3. Bruce DA, Alavi A, Bilaniuk L, Dolinskas C, Obrist W, Uzzell B: Diffuse cerebral swelling following head injuries in children: the syndrome of "malignant brain edema." J Neurosurg 54: $170-178,1981$

4. Eismont FJ, Clifford S, Goldberg M, Greene B: Cervical sagittal spinal canal size in spinal injury. Spine 9:663-666, 1984

5. Haymaker W, Woodhall B: Peripheral Nerve Injuries: Principles of Diagnosis, ed 2. Philadelphia: WB Saunders, 1953, pp 75,229

6. Keats TE: Atlas of Normal Roentgen Variants that May Simulate Disease, ed 6. St. Louis: Mosby, 1996, pp 208

7. Ladd AL, Scranton PE: Congenital cervical stenosis presenting as transient quadriplegia in athletes. J Bone Joint Surg Am 68: 1371-1374, 1986

8. McCrory PR, Berkovic SF: Second impact syndrome. Neurology 50:677-683, 1998

9. Pickles W: Acute general edema of the brain in children with head injuries. New Eng J Med 242:607-611, 1950

10. Saunders RL, Harbaugh RE: The second impact in catastrophic contact-sports head trauma. JAMA 252:538-539, 1984

11. Schneider RC: Head and Neck Injuries in Football: Mechanisms, Treatment, and Prevention. Baltimore: Williams \& Wilkins, 1973, pp 99, 173, 174

12. Torg JS, Corcoran TA, Thibault LE, Pavlov H, Sennett BJ, Naranja RJ Jr, et al: Cervical cord neurapraxia: classification, pathomechanics, morbidity, and management guidelines. J Neurosurg 87:843-850, 1997

Part of this material was presented at a seminar on "Head and Neck Football Injuries" at the annual meeting of the Congress of Neurological Surgeons in Boston, Massachusetts, in October, 1999.

Manuscript received August 3, 2006.

Accepted in final form September 7, 2006.

Address reprint requests to: G. Robert Nugent, M.D., Department of Neurosurgery, The Robert C. Byrd Health Sciences Center of West Virginia University, Morgantown, West Virginia 26506. email: gnugent@hsc.wvu.edu. 